\title{
Gasserian ganglion radiofrequency thermoablation in a patient with aneurysm of the anterior communicating artery -A case report-
}

Received March 2, 2021

Revised April 27, 2021

Accepted April 30, 2021

\section{Corresponding author}

Ji Hee Hong, M.D., Ph.D.

Department of Anesthesiology and

Pain Medicine, Keimyung University

Dongsan Hospital, 1035 Dalgubeol-

daero, Dalseo-gu, Daegu 42601,

Korea

Tel: 82-53-258-7767

Fax: 82-53-258-6288

E-mail: swon13@daum.net

\section{Yong Ho Lee, Ji Hee Hong, and Hye Kyung Shin}

Department of Anesthesiology and Pain Medicine, Keimyung University Dongsan Hospital, Daegu, Korea

Background: Gasserian ganglion radiofrequency thermoablation is a good treatment option for the management of pain in trigeminal neuralgia. We report a case in which the patient of trigeminal neuralgia combined with anterior cerebral artery aneurysm was treated successfully by gasserian ganglion thermoablation without any complication.

Case: An 85-year-old female presenting with electric shock like sensation in the gum and molar teeth was diagnosed as trigeminal neuralgia. Carbamazepine medication and trigeminal nerve blockade relieved her pain partially, but severe side effects of carbamazepine occurred. Magnetic resonance angiography of the brain showed saccular aneurysm in inferior aspect of the anterior communicating artery. Gasserian ganglion thermoablation under sedation anesthesia using nicardipine was performed carefully without any rupture of the cerebral aneurysm.

Conclusions: Gasserian ganglion thermoablation could be performed safely in a patient with cerebral aneurysm without any inadvertent event.

Keywords: Gasserian ganglion thermoablation; Nicardipine; Saccular aneurysm; Trigeminal neuralgia; Vital sign monitoring.
Patients of trigeminal neuralgia (TN) present a sudden severe, electric shock like, stabbing, and recurrent pain in the division area of the trigeminal nerve. The facial pain mostly demonstrates unilateral location with a cutaneous trigger zone. The peak age of onset is 50 to 60 years old. TN shows higher prevalence in women, ranging from $0.03-0.3 \%[1,2]$. Although the reported incidence of TN is low, the severe and disabling pain nature of TN significantly affect the quality of life with resultant psychologic distress, and may even lead to suicide [3].

Carbamazepine is known to demonstrate an excellent pain relief for TN. However, $10 \%$ of TN shows minimal re- sponse to oral medication. In addition, various side effects of carbamazepine including dizziness, loss of coordination, vertigo, and liver toxicity limit the popular use of such medication [4]. Various effective treatments including microvascular decompression (MVD), radiofrequency thermoablation, glycerol rhizolysis and percutaneous balloon microcompression are useful options for such intractable cases. Among such procedures, radiofrequency thermoablation demonstrates an excellent treatment outcome with a success rate of $80 \%$ to $97 \%$, although it shows reoccurrence in some of TN patients $[5,6]$.

For the diagnosis of $\mathrm{TN}$, characteristic clinical feature is

This is an Open Access article distributed under the terms of the Creative Commons Attribution Non-Commercial License (http://creativecommons.org/licenses/by-nc/4.0) which permits unrestricted non-commercial use, distribution, and reproduction in any medium, provided the original work is properly cited.

Copyright (C) the Korean Society of Anesthesiologists, 2021 
most important. With such clinical feature, magnetic resonance imaging (MRI) study of the brain is also recommended to rule out secondary $\mathrm{TN}[4,7]$.

We encountered a patient with severe gum and lower molar teeth pain of TN who was confirmed with anterior communicating artery saccular aneurysm by MRI and MR angiography (MRA). Here, we report this case as gasserian ganglion thermoablation was performed safely without any rupture of the saccular aneurysm. Before the preparation of this case report, written informed consent for publication was obtained from the patient.

\section{CASE REPORT}

An 85-year-old female with a height of $152 \mathrm{~cm}$ and a weight of $52 \mathrm{~kg}$, who presented severe left-sided lower teeth and gum pain, visited our pain clinic. Severe pain started 2 years ago. Her pain showed abrupt onset and termination with an electric shock like sensation. Duration of pain was within 1-2 min. Pain was usually provoked by washing face, brushing teeth, eating and mouth opening. Initially, she was evaluated at a dental clinic because her main symptom was in gum, lower molar teeth and mandibular area. Since, any problem was not found during dental examination, she was referred to the neurology department. She was diagnosed as TN at the neurology department, and medication was started with gabapentin $900 \mathrm{mg} /$ day, celecoxib $200 \mathrm{mg} /$ day and amitriptyline $10 \mathrm{mg} /$ day with minimal responses. Oral intake was very poor due to inadequate pain treatment. Her underlying disease was diabetes mellitus and hypertension.

According to her characteristic pain nature described above, we assessed her as TN. Carbamazepine $200 \mathrm{mg} /$ day was prescribed since she was not taking such medication previously. In addition, ultrasound-guided mental nerve blockade was performed with $0.2 \%$ ropivacaine $0.5 \mathrm{ml}$ using a hockey stick probe (Logiq S8, GE Healthcare, USA) (Fig. 1). MRI and MRA were ordered to rule out possible secondary $\mathrm{TN}$, and to evaluate any vascular anomaly or vascular compression of the trigeminal nerve.

Mental nerve blockade was performed twice with one week interval and carbamazepine medication was maintained for 16 days. She showed mild pain relief in lower teeth and gum after initiation of medication with mental nerve blockade. However, she demonstrated severe general weakness, dizziness, vertigo and poor oral intake. We assumed such symptoms to be side effects of carbamazepine. When carbamazepine medication was stopped, various symptoms observed after medication disappeared. Although partial pain relief was achieved even after termination of carbamazepine, maintaining normal daily life was impossible due to the reoccurrence of pain.

Gasserian ganglion thermoablation was planned to relieve intractable pain. Her MRI demonstrated mild brain atrophy, but otherwise normal findings. However, MRA showed saccular aneurysm $(5.1 \mathrm{~mm} \times 2.4 \mathrm{~mm})$ in the inferior aspect of the anterior communicating artery (Fig. 2). Due to the risk of abrupt rupture of the aneurysm during the procedure of gasserian ganglion thermoablation, we recommended delayed thermoablation after performing cerebral angiography or coiling. However, cerebral angiography or coiling was not possible until at least 3 weeks later due to overloaded schedules. Patient's intractable pain and poor general condition were too severe to wait for 3 weeks.

After explaining of possible rupture of the cerebral aneurysm, gasserian ganglion thermoablation was performed carefully under intravenous sedation anesthesia.

Before the procedure of thermoablation, light sedation was maintained with intravenous midazolam at $0.02 \mathrm{mg} / \mathrm{kg}$ and sufentanil $5 \mu \mathrm{g}$, so that the patient could respond properly during electrical stimulation. Patient was monitored with electrocardiography, blood pressure, and pulse oximetry. Facial mask was applied to supply oxygen ( $3 \mathrm{~L} / \mathrm{min})$. During thermoablation, we paid special attention to the changes of blood pressure, to minimize the risk of rupture of cerebral aneurysm. Her initial systolic blood pressure and heart rate was $155 \mathrm{mmHg}$ and 76 beats/min, respectively. Patient lay in the supine position with neck slightly extended, and chin up. The

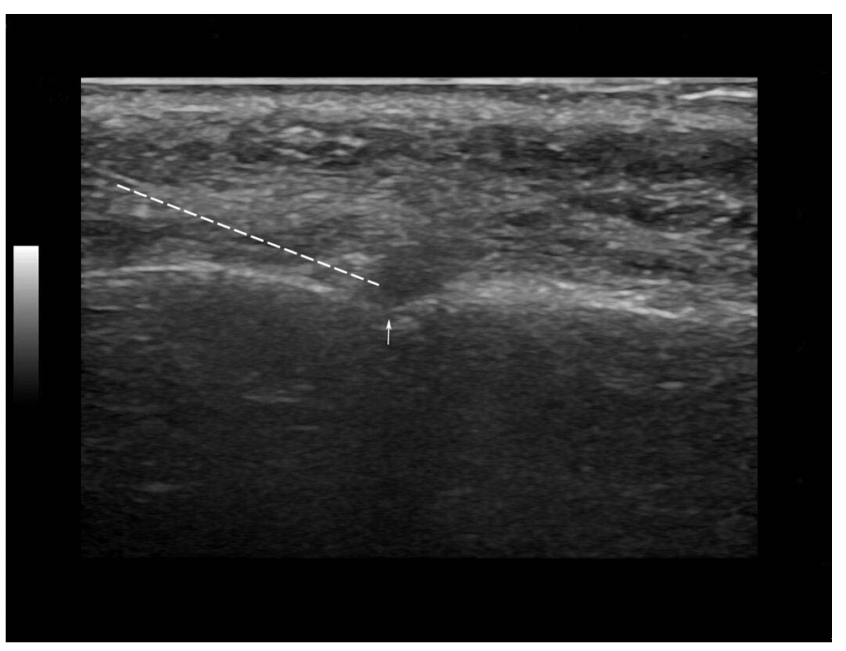

Fig. 1. Ultrasound guided mental nerve blockade. Dotted line and arrow indicate needle trajectory and mental foramen, respectively. 


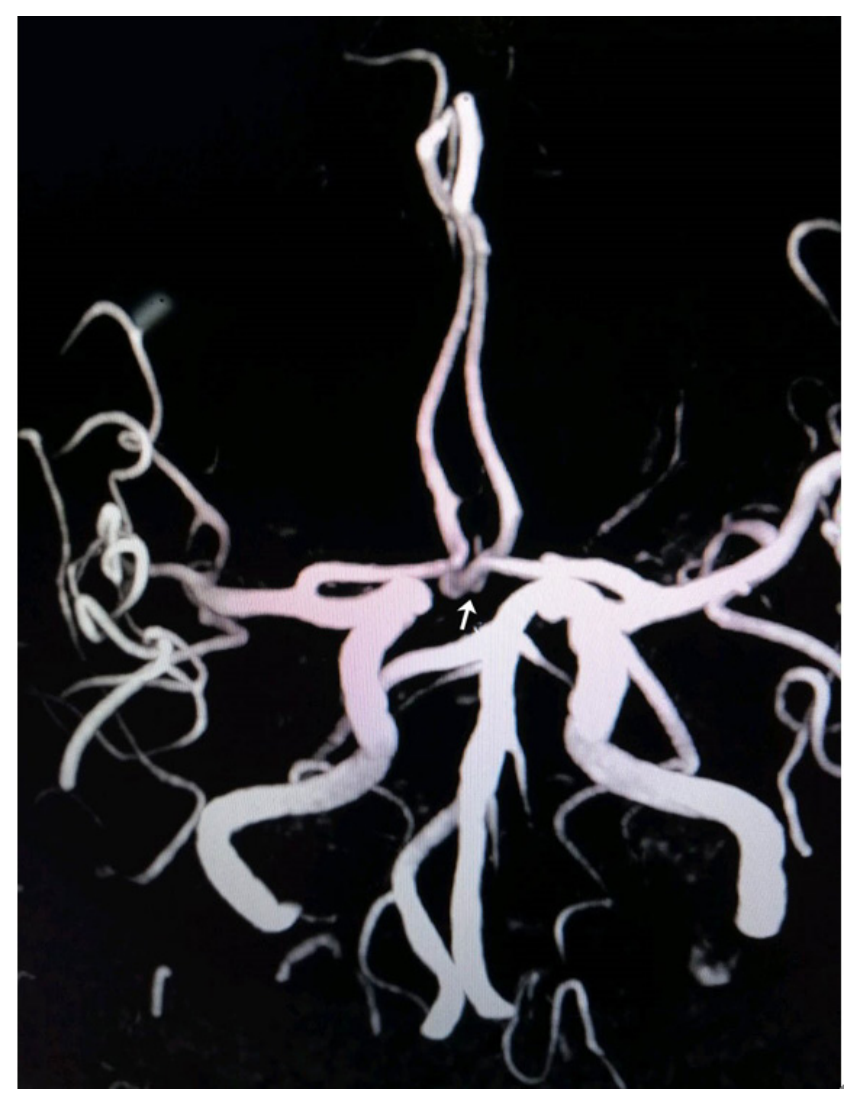

Fig. 2. Magentic resonance angiography of brain showing saccular aneurysm (arrow) in inferior aspect of the anterior communicating artery $(5.1 \mathrm{~mm} \times 2.4 \mathrm{~mm})$.
C-arm was tilted caudally 30 to 35 degrees, and rotated to the left side 20 to 25 degrees obliquely, to visualize the foramen ovale (FO). Skin entry was done at $2-3 \mathrm{~cm}$ lateral to the angle of the mouth. A radiofrequency cannula of 22-gauge, $10-\mathrm{cm}$, and $2 \mathrm{~mm}$ active tip was used. After confirming the clear visualization of the FO, the cannula was inserted in a coaxial manner into the fluoroscopic beam towards the FO.

When the cannula reached just in front of the FO in lateral view, nicardipine $1 \mathrm{mg}$ was injected to prevent abrupt increase of blood pressure. Systolic blood pressure before and after puncture of FO was $145 \mathrm{mmHg}$ and $135 \mathrm{mmHg}$, respectively.

After puncturing of the FO, the cannula was advanced 2-3 $\mathrm{mm}$ further and the location of cannula tip was confirmed. Once the cannula position was verified with $\mathrm{C}$-arm, the electrical stimulation of $0.1 \mathrm{~V}$ at $50 \mathrm{~Hz}$ frequency was in concordance with the location of the pain. The final position of the cannula tip was modified minutely, according to the effect of the stimulation (Fig. 3A, B). After successful concordant electrical stimulation in the mandibular area, radiofrequency was performed at $70^{\circ} \mathrm{C}$ for 60 seconds for one time. Just after finishing radiofrequency procedure, vital sign was stable except for the sinus tachycardia (112 beats/min). Patient was observed in the recovery area with vital sign monitoring. After taking a rest for an hour, she was in full awake state with
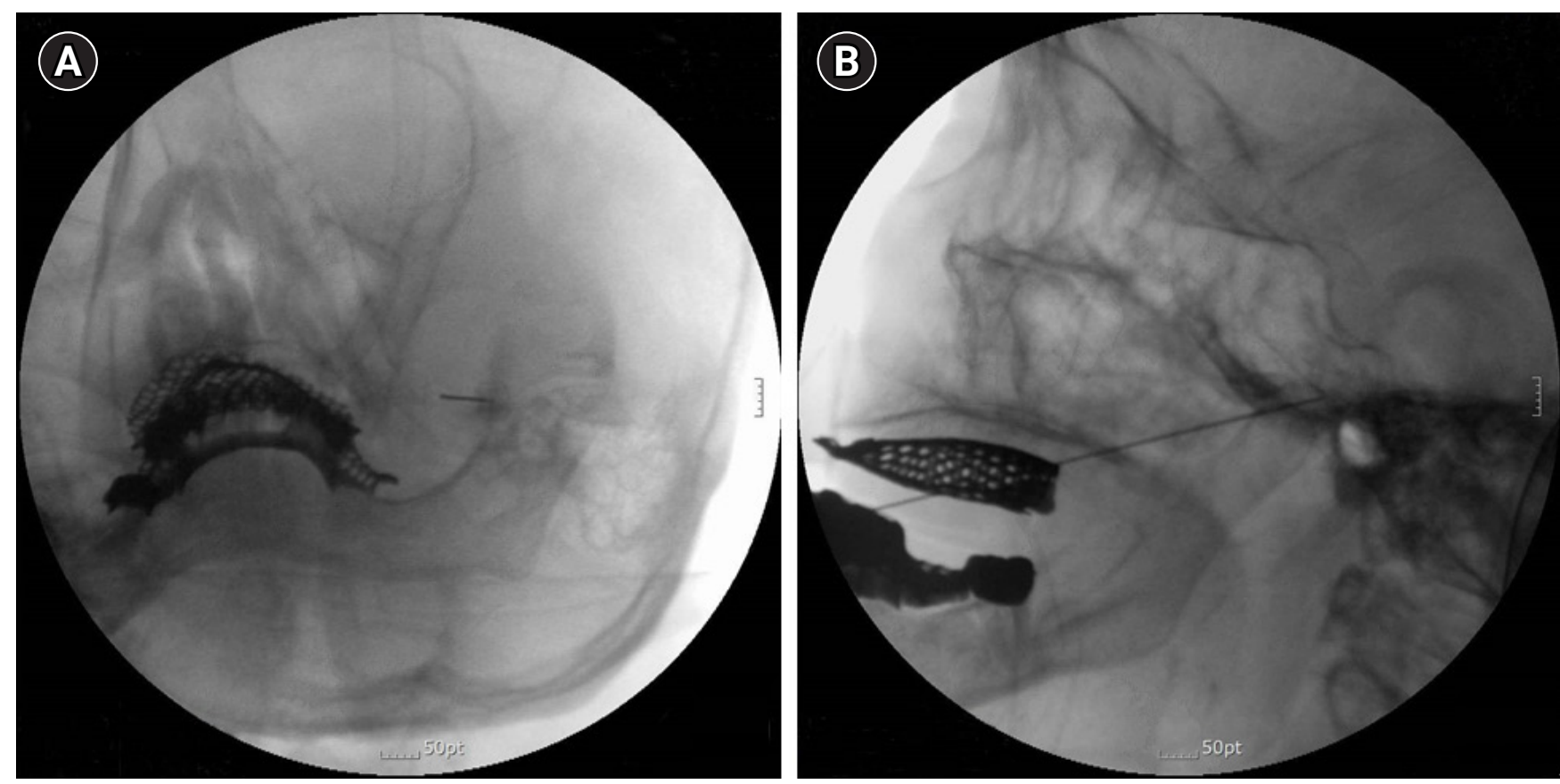

Fig. 3. Oblique (A) and lateral view (B) when the cannula tip reached the final location of mandibular division of the gasserian ganglion after electrical stimulation. 
stable vital sign.

She visited our pain clinic 2 weeks later, and her pain had disappeared completely with mild hypoesthesia around the chin.

\section{DISCUSSION}

Gasserian ganglion thermoablation in a patient with cerebral artery aneurysm is very challenging, due to the unexpected risk of aneurysm rupture. Cerebral aneurysm is found dominantly at artery bifurcations and hemodynamic instability or stresses play the most important role in the initiation, development, and rupture of aneurysm [8]. Hence, the risk of rupture of cerebral artery aneurysm should be assessed since unwanted hemodynamic responses during gasserian ganglion thermoablation might be encountered frequently $[9,10]$. Among procedure steps of thermoablation, FO puncture led to significant increase in heart rate $(42 / 48$, $88 \%$ ) and mean arterial pressure (48/48, $100 \%)$. Also, the heating stimulation of radiofrequency thermocoagulation obviously increased the mean arterial pressure and heart rate [9]. According to previous reports $[9,10]$ and our past experiences of thermoablation, we considered the moment of FO puncture and heating of gasserian ganglion would be the most critical event of unwanted increase in arterial pressure. Therefore, antihypertensive medication of nicardipine $1 \mathrm{mg}$ was injected just before puncturing of the FO to prevent abrupt increase of arterial pressure. As a result, the procedure was finished safely maintaining stable hemodynamics.

For antihypertensive medication, nicardipine was chosen because it shows fast onset of antihypertensive action with reflex increase in heart rate [11]. We thought that reflex increase in the heart rate of nicardipine would be beneficial, since sudden bradycardia can be found during gasserian ganglion thermoablation owing to trigeminocardiac reflex. Trigeminocardiac reflex can be found during percutaneous procedure to treat trigeminal neuralgia. It presents transient bradycardia or even sinus arrest $[10,12]$. Our patient did not show any bradycardia during the procedure.

Neurovascular conflict is the leading cause of TN, accounting for $80-90 \%$ of cases, although some patients of TN do not present any neurovascular conflict. An aberrant loop of artery or vein close to the trigeminal nerve root can result in such neurovascular conflict. The most common vessel resulting in neurovascular conflict is superior cerebellar artery (66-88\%) and less often, the anterior inferior cerebellar artery $(7.5-25 \%)[7,13]$. Although it is not frequent, aneurysm of the posterior cerebral artery can result in symptom of TN [13]. Similarly, giant posterior communicating artery aneurysm projecting to posterior fossa caused $\mathrm{TN}$ and surgical clipping of the aneurysm completely resolved severe facial pain [14]. We do not think that the anterior communicating artery aneurysm of this patient resulted in the symptom of $\mathrm{TN}$, since the trigeminal nerve and Meckel's cave are located in the posterior cranial fossa.

For patients with TN refractory to medical therapy, gasserian ganglion thermoablation, gamma knife, and MVD may be considered. In younger patients of TN, MVD is generally accepted as the treatment of choice. Previous studies indicated that MVD provided improved quality of life with long term pain relief. However, for elderly patients, gasserian ganglion thermoablation is preferred over MVD due to the increased morbidity and mortality associated with MVD [15].

The recurrence rate of thermoablation is $15 \%$ after 1 year of thermoablation, while MVD shows $8.4 \%$ after 2 years of surgery $[16,17]$.

For the purpose of identifying secondary TN due to compression by tumor or other mass lesion, brain MRI is strongly recommended in $\mathrm{TN}$ [7]. In pain clinics of our institution, we recommend MRI and MRA for better workup of other possible vascular lesion. In this case, if we did not evaluate with MRA, gasserian ganglion thermoablation might be performed without knowing the existence of cerebral aneurysm. The importance of MRA in patients of TN should be evaluated, since cerebral aneurysm itself might be a cause of TN. Also, by knowing the existence of cerebral aneurysm previously, we can take a step to minimize the hemodynamic changes during thermoablation.

Intravenous sedation anesthesia with vital sign monitoring should always be performed during gasserian ganglion thermoablation since this procedure is very painful and challenging. Propofol or midazolam as anxiolytic medications and opioids are used for sedation anesthesia [5,9]. If a patient combines $\mathrm{TN}$ with a cerebral aneurysm, the process of careful vital sign monitoring is an inevitable step.

In conclusion, we could perform gasserian ganglion thermoablation in a patient with anterior communicating artery aneurysm safely with careful vital sign monitoring using antihypertensive medication.

\section{CONFLICTS OF INTEREST}

No potential conflict of interest relevant to this article was reported. 


\section{DATA AVAILABILITY STATEMENT}

All data generated or analyzed during this study are included in this published article. This is a case report.

\section{AUTHOR CONTRIBUTIONS}

Writing - original draft: Yong Ho Lee, Ji Hee Hong. Writing review \& editing: Yong Ho Lee, Ji Hee Hong. Investigation: Yong Ho Lee, Ji Hee Hong, Hye Kyung Shin. Supervision: Ji Hee Hong.

\section{ORCID}

Yong Ho Lee, https://orcid.org/0000-0003-2545-7819

Ji Hee Hong, https://orcid.org/0000-0002-6679-3088

Hye Kyung Shin, https://orcid.org/0000-0001-6529-5274

\section{REFERENCES}

1. De Toledo IP, Conti Réus J, Fernandes M, Porporatti AL, Peres MA, Takaschima A, et al. Prevalence of trigeminal neuralgia: a systematic review. J Am Dent Assoc 2016; 147: 570-6.e2.

2. Huibin Q, Jianxing L, Guangyu H, Dianen F. The treatment of first division idiopathic trigeminal neuralgia with radiofrequency thermocoagulation of the peripheral branches compared to conventional radiofrequency. J Clin Neurosci 2009; 16: $1425-9$,

3. Tölle T, Dukes E, Sadosky A. Patient burden of trigeminal neuralgia: results from a cross-sectional survey of health state impairment and treatment patterns in six European countries. Pain Pract 2006; 6: 153-60.

4. Al-Quliti KW. Update on neuropathic pain treatment for trigeminal neuralgia. The pharmacological and surgical options. Neurosciences (Riyadh) 2015; 20: 107-14.

5. Kanpolat Y, Savas A, Bekar A, Berk C. Percutaneous controlled radiofrequency trigeminal rhizotomy for the treatment of idiopathic trigeminal neuralgia: 25-year experience with 1,600 patients. Neurosurgery 2001; 48: 524-32; discussion 532-4.

6. Bozkurt M, Al-Beyati ES, Ozdemir M, Kahilogullari G, Elhan $\mathrm{AH}$, Savas A, et al. Management of bilateral trigeminal neuralgia with trigeminal radiofrequency rhizotomy: a treatment strategy for the life-long disease. Acta Neurochir (Wien) 2012; 154: 785-91; discussion 791-2.

7. Love S, Coakham HB. Trigeminal neuralgia: pathology and pathogenesis. Brain 2001; 124(Pt 12): 2347-60.

8. Zhang XJ, Gao BL, Hao WL, Wu SS, Zhang DH. Presence of anterior communicating artery aneurysm is associated with age, bifurcation angle, and vessel diameter. Stroke 2018; 49: 341-7.

9. Meng Q, Zhang W, Yang Y, Zhou M, Li X. Cardiovascular responses during percutaneous radiofrequency thermocoagulation therapy in primary trigeminal neuralgia. J Neurosurg Anesthesiol 2008; 20: 131-5.

10. Cha ST, Eby JB, Katzen JT, Shahinian HK. Trigeminocardiac reflex: a unique case of recurrent asystole during bilateral trigeminal sensory root rhizotomy. J Craniomaxillofac Surg 2002; 30: 108-11.

11. Wallin JD, Cook ME, Blanski L, Bienvenu GS, Clifton GG, Langford $\mathrm{H}$, et al. Intravenous nicardipine for the treatment of severe hypertension. Am J Med 1988; 85: 331-8.

12. Qin Q, Wang Y. Recurrent trigeminocardiac reflex in percutaneous balloon compression for trigeminal neuralgia: a case report. Medicine (Baltimore) 2020; 99: e22467.

13. Dzierżanowski J, Słoniewski P. Trigeminal neuralgia caused by aneurysm of the posterior cerebral artery: a case description and the analysis of anatomical variety of vascular complex in the root entry zone of trigeminal nerve. Folia Morphol (Warsz) 2014; 73: 224-8.

14. Simonet C, Arrese I, Sarabia R. Giant posterior communicating artery aneurysm presenting as trigeminal neuralgia. J Stroke Cerebrovasc Dis 2016; 25: 2243-6.

15. Lopez BC, Hamlyn PJ, Zakrzewska JM. Systematic review of ablative neurosurgical techniques for the treatment of trigeminal neuralgia. Neurosurgery 2004; 54: 973-82; discussion 982-3.

16. Zheng S, Li X, Li R, Yang L, He L, Cao G, et al. Factors associated with long-term risk of recurrence after percutaneous radiofrequency thermocoagulation of the gasserian ganglion for patients with trigeminal neuralgia: a multicenter retrospective analysis. Clin J Pain 2019; 35: 958-66.

17. Zheng JH, Sun K, Zhang HT, Xie YJ, Wang-Yang LX, Chen HY, et al. A study on the recurrence rate of trigeminal neuralgia after MVD and the related factors. J Neurol Surg B Skull Base 2020; 81: 572-8. 